\title{
Transforming Chinese Traditional Patterns into Three-dimensions and Applying Them to Modern Design
}

\author{
YU QIAN \\ City College of WUST, Wuhan, China \\ qy0618@126.com
}

Keywords: Chinese Traditional Patterns, Product Design, Meaning, Shape

\begin{abstract}
For culture continuity and design innovations, the author researched how to apply three-dimensional traditional patterns to product design. This paper introduced the evolution and manifestations of Chinese traditional patterns; it discussed methods of and principles in making them three-dimensional. Through surface enclosure, increasing the thickness and image restoration, their external form and internal implication can be well integrated. By simplifying traditional patterns, combined with characteristics of the times and individuality, this will help to meet the aesthetic requirements of modern society.
\end{abstract}

\section{Introduction}

Chinese traditional patterns have a long history and diverse forms. They come from the life and work of the people; they are accumulated through generations of traditional culture. Inheriting and innovatively applying traditional cultural elements to modern design has become an unfailing subject. In product design, recreating traditional patterns is conducive to the construction of traditional Chinese culture; it also has great practical significance for improving the artistic taste of products and the people.

\section{Evolution of Traditional Patterns}

In the long stream of Chinese history, traditional patterns have been changing in contents, forms and cultural charm, which have witnessed the evolution of the nation. In the Neolithic Age, painted potteries had vivid, beautiful, classical and bold patterns, with animals, plants, men, and geometrics as the main contents, reflecting ancestors' totemism and instinct to pursue beauty. Symmetrical and balanced patterns in the Bronze Age were both bold and fine. The images of monsters and clouds, watermarks, geometrics showed the solemnity and mystery of bronze. In Qin and Han dynasties, patterns displayed tales, myths and legends with animals and people. They were simple and intricate, vivid, contrasting, and had a high artistic value. With the economic and cultural prosperity, Tang Dynasty had broader subjects, flowers and birds having been the main themes of this period. Patterns of Baoxiang flowers (a composite design which was a combination of different flowers), floral, flower sprays, curly grass, peonies, lotus flowers were passed down as traditional Chinese models. The decorative style was lively, realistic, and magnificent. Patterns of Qing Dynasty tended to be secular, and the images were detailed and delicate, often with flashy features. ${ }^{[1]}$

In all kind s of patterns, folk designs were rather unique and distinctive in development, in which there was a wide range of rich content. The most prominent were embroidery, paper cutting, shadow play, stone carvings, wood carvings and themes which included animals, people, flowers, birds, fish and insects. Homonyms, analogies, symbols and metaphors and other techniques were often adopted, giving rich meanings to the designs and reflecting people's good wish and pursuit for a better life.

Throughout the course of development of traditional patterns, we can see its manifestation was mostly two-dimensional, such as the designs on textiles, paper cuttings, wood carvings, or stone carvings. ${ }^{[2]}$ When traditional patterns are used in modern products, they appear as enhancing decorations. And restricted by the shape of products, the pattern may adapt to a different form of organization, such as separate patterns, situational patterns, radiating patterns and so on. In modern 
industry, traditional patterns provide important inspiration and guidance for clothing, packaging and furniture design. Likewise, they repeatedly appear in product design, and there have been many morphological beautiful works with rich implication.

\section{Methods of Transforming Traditional Patterns into Three-dimensions}

Study on transforming traditional patterns to three-dimensions will be imperative to the traditional cultural heritage and open up a broad path of development, keeping it alive. Traditional patterns provide products with rich meanings, and help to form the unique national characteristics. In addition, the Chinese traditional patterns were characterized by that "patterns must intentionally have auspicious features".[3] Therefore, based on the semantics conveyed in product features, they must also have the ability to convey the unique, traditional patterns with auspicious meanings. Transforming traditional patterns to three-dimensions is the inheritance and innovation of traditional culture. The methods are as follows:

\section{Flat or Curved Enclosure.}

Multiple flat planes can be enclosed or a flat plane can be curved, and take on three-dimensional effect. Traditional patterns were originally two-dimensional, by altering the shape of a flat plane and the patterns on it, a variety of geometrics or free shapes will be formed. This method is applicable to some Chinese lamps, furniture and containers. In reality, three-dimensional effect may be achieved by painting, drawing, pasting patterns directly on products' surface or by carving into and hollowing out the surface. This approach retains the two-dimensional characteristics of the traditional patterns, borrowing the three-dimensional shape of the product as the substrate, so the design is relatively simple, a rich visual effect can be achieved as long as three-dimensional shape of the product is reasonable. For example, in the following design of modern Chinese lamps (Fig.1), the traditional radiating patterns are put onto the four sides as decorations, which gives people a three-dimensional feeling.

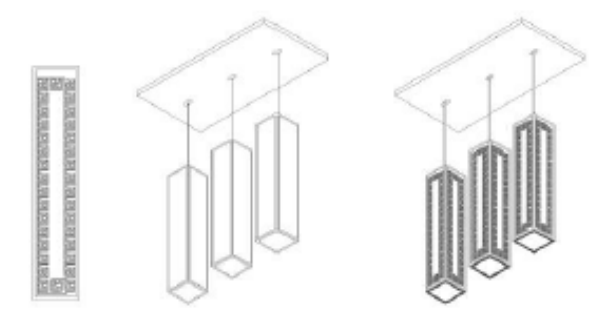

Fig. 1. Modern lamp with Chinese traditional patterns

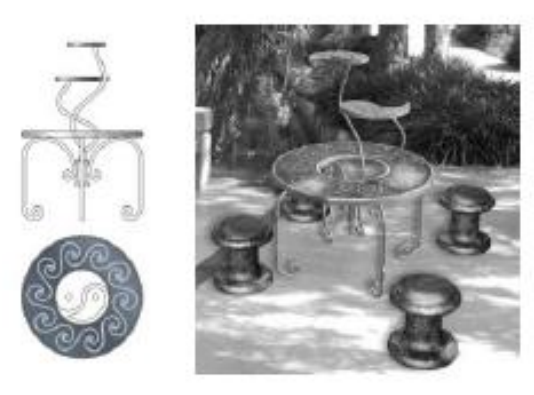

Fig.2. Outdoor stone table and stone stools 


\section{Increasing Thickness.}

This method, taking the traditional patterns as basic elements, reaches the three-dimensional effect by increasing patterns' thickness. It is comparable to the working principles of 3D designing software: a cube is formed by stretching; a rotary shade is formed through rotation; or a more complex shape is formed through mould-lofting. Thus, two-dimensional space is transformed to three-dimensional space. In actual design process, by flexible use of the above methods, combined with the aesthetic rules, an architect can syncretize traditional charm and formal beauty.

For instance, in Fig. 2, which is inspired by the traditional Taiji Diagram, thickness of the pattern is increased so that the original diagram appears to be three-dimensional. The design combines the massiveness of stone with the flexibility of metal, a typical material in modern design. The tabletop and stools are made of stone, and the supporting frame of the table is steel structure. Above the table, yin-yang fish patterns, which differ in size, are separated and have harmonious altitude difference. Steel frame spirals upwards, enhancing the motional effect. The supporting frame, the tabletop and stool tops all bear abstract image of Taiji Diagram. The overall design has balanced ratio relations and strong motions. It is a practical Landscape sketch, showing the reflection on and re-creation of traditional cultural essence. It is also a representative of the application of elements in the traditional patterns to modern design.

\section{Image Restoration.}

Traditional patterns stem from real life and serve the real life. Traditional Chinese patterns, having taken root in the ancient history and culture of China, carry a distinctive nationality and regional traits, some folk patterns also have a strong sense from daily life. Patterns such as dragon, phoenix, peonies, lotus flowers, curly grass, etc., emphasize the completeness and beauty of forms; they must be tangled together and echo each other in the overall design that is featured by symmetrical and balanced arrangement. ${ }^{[4]}$ In modern design, an architect can partially or wholly restore the image of the concrete or abstract symbols to make it become three-dimensional without disrupting the overall organizational pattern, which, after all, is a good method of design. Take jewelry design for example, designers can borrow traditional patterns, like animals or flowers, restore the image and put them back into three-dimensions that resemble the image that people see in real life. ${ }^{[5]}$

\section{Basic Principles of the Applying Three-dimensional Traditional Patterns to Product Design}

To transform from the two-dimensional to the three-dimensional, the aforesaid methods will be helpful but those alone aren't adequate. In order to perfectly integrate formal beauty with functionality, combination and transfiguration will be needed to further process the pattern. Due to the particularity of traditional patterns, their three-dimensional design should follow some basic principles:

\section{Simplification.}

In the evolution of traditional patterns, they were influenced by the thoughts of various historical periods and became more and more detailed and complex, especially when it comes to the modern times, they are supreme intricate, which could not meet the needs of the modern aesthetic taste. Modern patterns must be simple and brief, and the overall feature of modern products is concise and clear. So, traditional patterns, when applied to modern products, should be simplified. Image generalization, partial adoption, abstraction are all means of simplification, which is a basic way to sort out and rearrange traditional patterns. Generalization does not mean omission or loss, but a refining and a re-creation of art. In other words, that is to grasp the general profile and essence of the complicated patterns, drop the cumbersome parts and details, highlight the chief features, so that the patterns are purified, yet maintain the decorative beauty of the original patterns. ${ }^{[6]}$ But it is essential to note that, first of all, functional characteristics of the product should be clarified. As the saying goes, "patterns are the carrier of products, products are made on patterns." Transforming traditional patterns into three-dimensional is an innovation in form, and form is second to function. Therefore, to 
simplify traditional patterns, designers should start with a generalization of the product's function and then make appropriate transfiguration.

\section{Integrating Form and Meaning.}

Traditional patterns have not only varied forms, but also rich meanings. The themes expressed in them are "Good Luck", "Dreams Coming True" "Living Peacefully", "Happiness", "Wealth", "Long Life", "Joy", which are the eternal topics for thousands of years. They reveal Chinese people's wishes for and pursuit of everything that is good, reflecting the pure and kind nature of this nation. From the evolutionary course of traditional patterns, it can be found that many of them are enduring and have exuberant vitality because they carry behind them auspicious meanings. ${ }^{[7]}$ In addition to meeting the needs of practicality, products should also suit consumers' aesthetic taste. So, it is crucial to understand their auspicious meanings in choosing traditional patterns. Form is the external; meaning is the internal, form and meaning in combination are to make the products complete. In the inheritance and development of traditional patterns, to borrow the form is the most primitive and simple step, the key and most significant step is to extend the meaning and convey the essence.

\section{Conforming with the Times and Individualized Tastes.}

The form and content of traditional patterns are always evolving with the passing of the times. While transforming them into three-dimensional patterns, architects must pay attention to the aesthetic requirements of the times and of individuals in modern society, and re-create the patterns; they must find out the local expressions in traditional patterns, understand profoundly the form and meanings, re-place them in the contemporary culture, meet the design theme, refer to the modern aesthetic characteristics, think rationally, understand deeply and perform boldly to achieve a combination of traditional elements and the spirit of the times, so that the new design will bear characteristics of the times, instead of simply copying or recovering the traditional style. Besides, uniqueness is another characteristic of modern products. ${ }^{[8]}$ In modern society, people express their personality by choosing products that can represent the quality of their life. Traditional patterns are diversified in shape and thus offer abundant material for the realization of individualized needs. At the same time, the unique ethnic characteristics and profound auspicious meaning of traditional patterns meet the taste of Chinese people, and are favorable to reflect the Chinese national character.

The design instruments, new materials, producing crafts of modern product pose new challenges to the development and heritage of traditional patterns. Similarly, the application of new materials and new technologies to traditional patterns creates distinctive characteristics of the times and broad space for development too.

\section{Summary}

Innovation is the driving force for the heritage and evolution of traditional culture. Recreation of the traditional patterns has the same function. Innovation happens in diverse forms, in addition to altering the design itself, expanding the application areas of the pattern being another significant means. In product design, besides being used for partial or overall decoration, traditional patterns can also be transformed into three dimensions through surface enclosure, increasing thickness and image restoration, thus becoming the product modeling. Architects should simplify the design in accordance with products' semantic functions so that the "design" and "meaning" is integrated, giving the products traditional cultural forms and rich cultural content. By this means, Chinese traditional patterns can be innovated in form and inherited in meaning. What's more, this inheritance and innovation must echo with the times and be closely linked with individual demands. Only in this way can the traditional patterns be accepted by modern people, kept alive and carried forward. 


\section{Acknowledgement}

This paper is a current result for the 2015 Hubei province educational science planning project entitled "The Teaching Model of National Folk Art Intervening the Cultivation of Innovative Ability of Students Majored in Environment Design” (project No.: 2015GB163).

\section{References}

[1] Shao-yuan Li, Graphic Design, Guangxi Fine Arts Publishing House, Nanning, 2009.

[2] Ling Zhong, Use of the Traditional Patterns and Functional Expressions of Modern Products, Packaging Engineering, 31 (2010) 38-41.

[3] Wei-cai Wen, Zhuan-yi Huang, On the Application of Auspicious Meanings of Traditional Patterns to Modern Chinese Lamps Design , Packaging Engineering, 31 (2010) 46-49.

[4] Jian-ping Ma, Traditional Patterns in Modern Arts and Design, Art Grand, 7 (2011) 150.

[5] Qiong Peng, Chinese Traditional Patterns and Modern Jewelry Design, Art and Design, 2 (2010) 198-200.

[6] Fang Ma, The Application of Traditional Patterns to Modern Furniture Design, The Art Sea, 5 (2011) 93-94.

[7] Tian Tian, The Infiltration of Traditional Patterns in Modern Design, Art Grand, 1 (2011) 124.

[8] Juan Guo, A New Exploration of the Teaching of Innovating Chinese Traditional Patterns , Decoration, 1 (2011) 120-121. 